\title{
A Quintom scenario with mixed kinetic terms
}

\author{
Emmanuel N. Saridakis ${ }^{1, \text { 田 }}$ and Joel M. Weller ${ }^{2, \text { t }}$ \\ ${ }^{1}$ College of Mathematics and Physics, Chongqing University of Posts and Telecommunications, \\ Chongqing, 400065, P.R. China \\ ${ }^{2}$ Department of Applied Mathematics, University of Sheffield, \\ Hounsfield Road, Sheffield S3 7RH, United Kingdom
}

\begin{abstract}
We examine an extension of the quintom scenario of dark energy, in which a canonical scalar field and a phantom field are coupled through a kinetic interaction. We perform a phase space analysis and show that the kinetic coupling gives rise to novel cosmological behaviour. In particular, we obtain both quintessence-like and phantom-like late-time solutions, as well as solutions that cross the phantom divide during the evolution of the Universe.

PACS numbers: 98.80.-k, 95.36.+x
\end{abstract}

\section{INTRODUCTION}

According to independent observational data, including measurements of type Ia supernovae [1], the Wilkinson Microwave Anisotropy Probe 2], the Sloan Digital Sky Survey [3], and X-ray observations [4], the Universe is undergoing a phase of accelerated expansion. Although the cosmological constant seems to be a simple and economic way to explain this behaviour [5], the extreme degree of fine-tuning associated with this means that it is attractive to consider other dark energy candidates. The dynamical nature of dark energy, at least at an effective level, can originate from various fields, such as a canonical scalar field (quintessence) [6] or a phantom field [7] (i.e. a scalar field with negative kinetic terms). Another possibility of considerable interest is the quintom scenario 8 -10], in which the acceleration is driven by a combination of quintessence and phantom fields. The quintom paradigm has the advantage of allowing the dark energy equation-of-state (EOS) parameter $\left(w_{\mathrm{DE}}\right)$ to cross the phantom divide during the evolution of the Universe, an intriguing possibility for which there is observational evidence from a variety of sources (see [10] for a review).

The aforementioned models offer a satisfactory description of the behaviour of dark energy and its observable features. However, the dynamical nature of dark energy in these scenarios leads to the "coincidence" problem, which many authors have sought to resolve by considering a coupling between dark energy and the other components of the Universe. Thus, various forms of interacting dark energy models have been constructed, including coupled quintessence [11] and interacting phantom models [12]. Similarly, the dynamical nature of dark energy makes it difficult to fulfil the observational requirement, $w_{\mathrm{DE}} \approx-1[13$, which has led to the introduction of additional scalar fields. Inspired by similar multi-field inflation [14] and assisted inflation constructions [15], various

*Electronic address: msaridak@phys.uoa.gr

${ }^{\dagger}$ Electronic address: app07jmw@shef.ac.uk forms of assisted dark energy models have been considered [16, 17].

One generalised class of the multi-field quintessence scenario allows for a mixing of the fields' kinetic terms [18] (see also [19]). Mixed kinetic terms appear also in the non-trivial scalar field models based on string theory constructions, studied in the framework of generalised multi-field inflation [20].

In the present work we aim to combine the advantages of the quintom scenario with those of assisted quintessence with mixed kinetic terms. Thus, we construct a quintom model in which the kinetic terms of the canonical and the phantom fields are mixed. Indeed, as we see, the model exhibits features characteristic of both quintessence and phantom models.

The plan of the work is as follows. In Sec. II we construct the quintom cosmological scenario with mixed kinetic terms and present the formalism for its transformation into an autonomous dynamical system. In Sec. III we perform the phase-space stability analysis and in Sec. IV we discuss the cosmological implications of the results. Finally, our conclusions are presented in Sec. V.

\section{QUINTOM SCENARIO WITH MIXED KINETIC TERMS}

Let us construct the quintom cosmological scenario with mixed kinetic terms. Throughout the work we consider a flat Robertson-Walker metric:

$$
d s^{2}=d t^{2}-a^{2}(t) \mathbf{d} \mathbf{x}^{2},
$$

with $a$ the scale factor.

We consider a model consisting of a canonical scalar field $\phi$, a phantom field $\sigma$ and a mixed kinetic term proportional to the parameter $\alpha$. Thus, the action, in units where $8 \pi G=1$ reads:

$$
\begin{array}{r}
S=\int d^{4} x \sqrt{-g}\left[\frac{R}{2}-\frac{1}{2} g^{\mu \nu} \partial_{\mu} \phi \partial_{\nu} \phi+\frac{1}{2} g^{\mu \nu} \partial_{\mu} \sigma \partial_{\nu} \sigma-\right. \\
\left.-\frac{\alpha}{2} g^{\mu \nu} \partial_{\mu} \phi \partial_{\nu} \sigma+V_{\phi}(\phi)+V_{\sigma}(\sigma)\right]+S_{M},
\end{array}
$$


with $V_{\phi}(\phi)$ and $V_{\sigma}(\sigma)$ the corresponding field potentials, and $S_{M}$ the action for the matter (dark plus baryonic) component of the Universe. We assume that the matter is described by a perfect fluid with energy density $\rho_{M}$, pressure $p_{M}$ and barotropic index $\gamma$. Thus, $p_{M}=(\gamma-1) \rho_{M}$ and the matter equation-of-state parameter is $w_{M}=\gamma-1$. Varying the action with respect to the metric yields the Friedmann equations

$$
\begin{aligned}
& H^{2}=\frac{1}{3}\left[\frac{1}{2} \dot{\phi}^{2}-\frac{1}{2} \dot{\sigma}^{2}+\right. \frac{\alpha}{2} \dot{\phi} \dot{\sigma}+ \\
&\left.+V_{\phi}(\phi)+V_{\sigma}(\sigma)+\rho_{M}\right], \\
& \dot{H}+\frac{3}{2} H^{2}=-\frac{1}{2}\left[\frac{1}{2} \dot{\phi}^{2}-\frac{1}{2} \dot{\sigma}^{2}+\frac{\alpha}{2} \dot{\phi} \dot{\sigma}-\right. \\
&\left.-V_{\phi}(\phi)-V_{\sigma}(\sigma)+p_{M}\right],
\end{aligned}
$$

while the evolution equations for the two fields read

$$
\begin{gathered}
\ddot{\phi}+3 H \dot{\phi}+\left(\frac{\partial V_{\phi}}{\partial \phi}+\frac{\alpha}{2} \frac{\partial V_{\sigma}}{\partial \sigma}\right)\left(1+\frac{\alpha^{2}}{4}\right)^{-1}=0, \\
\ddot{\sigma}+3 H \dot{\sigma}+\left(-\frac{\partial V_{\sigma}}{\partial \sigma}+\frac{\alpha}{2} \frac{\partial V_{\phi}}{\partial \phi}\right)\left(1+\frac{\alpha^{2}}{4}\right)^{-1}=0 .
\end{gathered}
$$

In quintom scenarios the dark energy is attributed to the combination of the two scalar fields. In the case at hand, from the Friedmann equations (3), (4) we straightforwardly read the dark energy density and pressure as

$$
\begin{aligned}
& \rho_{D E}=\frac{1}{2} \dot{\phi}^{2}-\frac{1}{2} \dot{\sigma}^{2}+\frac{\alpha}{2} \dot{\phi} \dot{\sigma}+V_{\phi}(\phi)+V_{\sigma}(\sigma) \\
& p_{D E}=\frac{1}{2} \dot{\phi}^{2}-\frac{1}{2} \dot{\sigma}^{2}+\frac{\alpha}{2} \dot{\phi} \dot{\sigma}-V_{\phi}(\phi)-V_{\sigma}(\sigma),
\end{aligned}
$$

and thus for the dark energy equation-of-state parameter we obtain

$$
w_{\mathrm{DE}} \equiv \frac{p_{D E}}{\rho_{D E}}=\frac{\frac{1}{2} \dot{\phi}^{2}-\frac{1}{2} \dot{\sigma}^{2}+\frac{\alpha}{2} \dot{\phi} \dot{\sigma}-V_{\phi}(\phi)-V_{\sigma}(\sigma)}{\frac{1}{2} \dot{\phi}^{2}-\frac{1}{2} \dot{\sigma}^{2}+\frac{\alpha}{2} \dot{\phi} \dot{\sigma}+V_{\phi}(\phi)+V_{\sigma}(\sigma)} .
$$

Finally, using (7), (8) and (5), (6), we can easily acquire the dark energy evolution equation in fluid terms, which takes the expected form

$$
\dot{\rho}_{D E}+3 H\left(\rho_{D E}+p_{D E}\right)=0 .
$$

We mention that in the case $\alpha=0$ the above scenario coincides with the usual quintom one [9].

\section{PHASE-SPACE ANALYSIS}

In order to perform the phase-space and stability analysis of the quintom model at hand, we have to transform the aforementioned dynamical system into its autonomous form [21 23]. This can be achieved by intro- ducing the auxiliary variables:

$$
\begin{array}{cc}
x_{\phi}=\frac{\dot{\phi}}{\sqrt{6} H}, & x_{\sigma}=\frac{\dot{\sigma}}{\sqrt{6} H}, \\
y_{\phi}=\frac{\sqrt{V_{\phi}(\phi)}}{\sqrt{3} H}, & y_{\sigma}=\frac{\sqrt{V_{\sigma}(\sigma)}}{\sqrt{3} H},
\end{array}
$$

together with $N=\ln a$. It is easy to see that for every quantity $F$ we acquire $\dot{F}=H \frac{d F}{d N}$. Using these variables, from (7) we can calculate the dark energy density parameter,

$$
\Omega_{D E} \equiv \frac{\rho_{D E}}{3 H^{2}}=x_{\phi}^{2}-x_{\sigma}^{2}+\alpha x_{\phi} x_{\sigma}+y_{\phi}^{2}+y_{\sigma}^{2},
$$

and from (9) the dark energy equation-of-state parameter,

$$
w_{\mathrm{DE}}=\frac{x_{\phi}^{2}-x_{\sigma}^{2}+\alpha x_{\phi} x_{\sigma}-y_{\phi}^{2}-y_{\sigma}^{2}}{x_{\phi}^{2}-x_{\sigma}^{2}+\alpha x_{\phi} x_{\sigma}+y_{\phi}^{2}+y_{\sigma}^{2}} .
$$

The final step is the consideration of specific potential forms. Following works on assisted quintessence 17] and assisted inflation [15] scenarios, together with various other cosmological models [21, 23, 24], we consider exponential potentials of the form,

$$
V_{\phi}(\phi)=e^{-\lambda \phi}, \quad V_{\sigma}(\sigma)=e^{-\mu \sigma} .
$$

Note that equivalently, but more generally, we could consider potentials satisfying $\lambda=-\frac{1}{\kappa V(\phi)} \frac{\partial V(\phi)}{\partial \phi} \approx$ const, $\mu=-\frac{1}{\kappa V(\sigma)} \frac{\partial V(\sigma)}{\partial \sigma} \approx$ const (for example this relation is valid for arbitrary but nearly flat potentials [25]). Finally, without loss of generality, in this work we consider $\lambda$ and $\mu$ to be positive, since the negative case alters only the direction towards which the fields roll, and can always be transformed into the positive case by changing the signs of the fields themselves.

Let us now perform the phase-space analysis of the model. In general, having transformed the cosmological system into its autonomous form $\mathbf{X}^{\prime}=\mathbf{f}(\mathbf{X})$, where $\mathbf{X}$ is the column vector constituted by the auxiliary variables, $\mathbf{f}(\mathbf{X})$ the corresponding column vector of the autonomous equations, and prime denotes a derivative with respect to $N=\ln a$, the critical points $\mathbf{X}_{\mathbf{c}}$ are extracted satisfying $\mathbf{X}^{\prime}=\mathbf{0}$. In order to determine the stability properties of these critical points we expand around $\mathbf{X}_{\mathbf{c}}$, setting $\mathbf{X}=\mathbf{X}_{\mathbf{c}}+\mathbf{U}$ with $\mathbf{U}$ the perturbations of the variables considered as a column vector. Up to first order we acquire $\mathbf{U}^{\prime}=\mathbf{Q} \cdot \mathbf{U}$, where the matrix $\mathbf{Q}$ contains the coefficients of the perturbation equations. Thus, for each critical point, the eigenvalues of $\mathbf{Q}$ determine its type and stability.

Following this method and using the auxiliary variables (11), the cosmological equations of motion (3), (4), (5) 
and (6) become

$$
\begin{aligned}
x_{\phi}^{\prime} & =-3 x_{\phi}+\sqrt{\frac{3}{2}}\left(1+\frac{\alpha^{2}}{4}\right)^{-1}\left(\lambda y_{\phi}^{2}+\frac{\alpha}{2} \mu y_{\sigma}^{2}\right)+x_{\phi} T \\
x_{\sigma}^{\prime} & =-3 x_{\sigma}-\sqrt{\frac{3}{2}}\left(1+\frac{\alpha^{2}}{4}\right)^{-1}\left(\mu y_{\sigma}^{2}-\frac{\alpha}{2} \lambda y_{\phi}^{2}\right)+x_{\sigma} T \\
y_{\phi}^{\prime} & =-\sqrt{\frac{3}{2}} \lambda x_{\phi} y_{\phi}+y_{\phi} T \\
y_{\sigma}^{\prime} & =-\sqrt{\frac{3}{2}} \mu x_{\sigma} y_{\sigma}+y_{\sigma} T
\end{aligned}
$$

where

$$
\begin{aligned}
T= & \frac{3}{2}\left[2 x_{\phi}^{2}-2 x_{\sigma}^{2}+2 \alpha x_{\phi} x_{\sigma}\right. \\
& \left.+\gamma\left(1-x_{\phi}^{2}+x_{\sigma}^{2}-\alpha x_{\phi} x_{\sigma}-y_{\phi}^{2}-y_{\sigma}^{2}\right)\right] .
\end{aligned}
$$

The critical points $\left(x_{\phi c}, x_{\sigma c}, y_{\phi c}, y_{\sigma c}\right)$ of the autonomous system (15) are obtained by setting the left hand sides of the equations to zero. The real and physically meaningful (i.e. corresponding to $y_{\phi}, y_{\sigma}>0$, and $\left.0 \leq \Omega_{D E} \leq 1\right)$ points are presented in Table [

The $4 \times 4$ matrix $\mathbf{Q}$ of the linearised perturbation equations of the system (15) is shown in the Appendix. Thus, for each critical point of Table $\Pi$ we examine the sign of the real part of the eigenvalues of $\mathbf{Q}$ to determine the type and stability of the point. In Table II we present the results of the stability analysis. In addition, for each critical point we calculate the values of $\Omega_{D E}$ [given by (12)] and of $w_{\mathrm{DE}}$ [given by (13)]. Finally, we mention that the labelling of the critical points follows the convention used in [18]. That is, since in a two-field system there are critical points in which the potential term of one of the fields is zero, the connection between these points is made more explicit. For instance, in the points $D 1$ and D2 (for which $\Omega_{D E}=1$ ) only one of the fields' potential terms contributes to the energy density. Note that in this model (unlike that in [18]) the solution satisfying $\mathbf{X}^{\prime}=\mathbf{0}$ with $y_{\phi}=0$ corresponding to $C 1$ is unphysical.

The stability of the critical points can be summarised as follows:

\section{- Point $A$}

This is a trivial solution, corresponding to the fluid dominated point $F$ in [8], where the kinetic and potential components of both the phantom and the quintessence fields are negligible. It exists for all values of $\alpha, \lambda$ and $\mu$. The eigenvalues are

$$
\begin{aligned}
& e_{1}=e_{2}=\frac{3}{2} \gamma>0, \\
& e_{3}=e_{4}=-\frac{3}{2}(2-\gamma)<0,
\end{aligned}
$$

so this is a saddle point.

\section{- Point $B$}

The two points indicated by the \pm are similar to point B in [18]. When $\alpha=0$ (point $K$ in [8]), there is a continuous locus of points that describes a hyperbola in the $\left(x_{\phi}, x_{\sigma}\right)$ plane. When $\alpha \neq 0$ the locus still describes a hyperbola, but the asymptotes are no longer orthogonal. The eigenvalues are

$$
\begin{aligned}
e_{1} & =3(2-\gamma)>0, \\
e_{2} & =0, \\
e_{3} & =3-\frac{5 \sqrt{6}}{4} \mu x_{\sigma} \\
& +\frac{3 \sqrt{6}}{4} \lambda\left[-\frac{1}{2} x_{\sigma} \alpha \pm \frac{1}{2} \sqrt{x_{\sigma}^{2}\left(\alpha^{2}+4\right)+4}\right], \\
e_{4} & =3+\frac{3 \sqrt{6}}{4} \mu x_{\sigma} \\
& -\frac{5 \sqrt{6}}{4} \lambda\left[-\frac{1}{2} x_{\sigma} \alpha \pm \frac{1}{2} \sqrt{x_{\sigma}^{2}\left(\alpha^{2}+4\right)+4}\right],
\end{aligned}
$$

where the \pm in $e_{3}$ and $e_{4}$ corresponds to the particular solution considered. $e_{1}$ is positive, therefore the point is unstable.

\section{- Point $D 1$}

This takes a similar form to point D1 in [18]. (The $y_{\sigma}$ value in the solution corresponding to $C 1$ in [18] has a factor of $\sqrt{\left(4+\alpha^{2}\right)(1-2 / \gamma)}$ so there are no real values of $\alpha$ for which the point exists.) In the standard quintom case $(\alpha=0), D 1$ is a phantomdominated point (point $P$ in the notation of $[8]$ ). In that case, as in the present one, the term under the square root in $y_{\sigma c}$ is positive and $\Omega_{D E}=1$, so the point exists for all values of the parameters. However, in the case at hand, the eigenvalues read

$$
\begin{aligned}
e_{1,2} & =-\frac{2 \mu^{2}}{\left(4+\alpha^{2}\right)}-3 \leq 0, \\
e_{3} & =-\frac{4 \mu^{2}}{\left(4+\alpha^{2}\right)}-3 \gamma \leq 0, \\
e_{4} & =-\frac{\mu(2 \mu+\alpha \lambda)}{\left(4+\alpha^{2}\right)},
\end{aligned}
$$

so the point is stable only if $(2 \mu+\alpha \lambda)>0$, which is trivially satisfied when $\alpha \geq 0$.

\section{- Point $\mathrm{C2}$}

The points C2 and D2 are similar to D1, in that only one of the fields' potential energy contributes to the total energy density; in $D 1$ this was the phantom field, while here it is the quintessence one. This means that there is both a scaling solution (C2) and a field-dominated solution (D2), for which $\Omega_{D E}=1$. The density parameter for C2 is $\Omega_{D E}=\frac{3 \gamma}{4 \lambda^{2}}\left(4+\alpha^{2}\right)$, so the condition $\Omega_{D E} \leq 1$ means that $\lambda \geq \sqrt{\frac{3 \gamma}{4}\left(4+\alpha^{2}\right)}$ must be satisfied if the point is to be physical. The eigenvalues are

$$
\begin{aligned}
e_{1} & =-\frac{3}{2}(2-\gamma)<0, \\
e_{2} & =\frac{3 \gamma}{4 \lambda}(2 \lambda-\alpha \mu), \\
e_{3,4} & =-\frac{3(2-\gamma)}{4} \\
\times & {\left[1 \pm \sqrt{\frac{6 \gamma^{2}\left(4+\alpha^{2}\right)+\lambda^{2}(2-9 \gamma)}{\lambda^{2}(2-\gamma)}}\right] . }
\end{aligned}
$$




\begin{tabular}{|c||c|c|c|c|}
\hline & $x_{\phi c}$ & $x_{\sigma c}$ & $y_{\phi c}$ & $y_{\sigma c}$ \\
\hline \hline $\mathrm{A}$ & 0 & 0 & 0 & 0 \\
\hline $\mathrm{B}$ & $-\frac{1}{2} x_{\sigma} \alpha \pm \frac{1}{2} \sqrt{x_{\sigma}^{2}\left(\alpha^{2}+4\right)+4}$ & $x_{\sigma}$ & 0 & 0 \\
\hline $\mathrm{D} 1$ & $\frac{\sqrt{6} \mu \alpha}{3\left(4+\alpha^{2}\right)}$ & $-\frac{2 \sqrt{6} \mu}{3\left(4+\alpha^{2}\right)}$ & 0 & $\frac{\sqrt{6}}{3\left(4+\alpha^{2}\right)} \sqrt{\left(4+\alpha^{2}\right)\left(6+\frac{3}{2} \alpha^{2}+\mu^{2}\right)}$ \\
\hline $\mathrm{C} 2$ & $\frac{\gamma \sqrt{6}}{2 \lambda}$ & $\frac{\alpha \gamma \sqrt{6}}{4 \lambda}$ & $\frac{\gamma \sqrt{6}}{4 \lambda} \sqrt{\left(4+\alpha^{2}\right)\left(\frac{2}{\gamma}-1\right)}$ & 0 \\
\hline $\mathrm{D} 2$ & $\frac{2 \sqrt{6} \lambda}{3\left(4+\alpha^{2}\right)}$ & $\frac{\sqrt{6} \lambda \alpha}{3\left(4+\alpha^{2}\right)}$ & $\frac{\sqrt{6}}{3\left(4+\alpha^{2}\right)} \sqrt{\left(4+\alpha^{2}\right)\left(6+\frac{3}{2} \alpha^{2}-\lambda^{2}\right)}$ & 0 \\
\hline $\mathrm{E}$ & $\sqrt{\frac{3}{2}} \frac{\gamma}{\lambda}$ & $\sqrt{\frac{3}{2}} \frac{\gamma}{\mu}$ & $\sqrt{\frac{3}{2}} \frac{\gamma}{\mu \lambda} \sqrt{\mu\left(\mu+\frac{\alpha}{2} \lambda\right)\left(\frac{2}{\gamma}-1\right)}$ & $\sqrt{\frac{3}{2}} \frac{\gamma}{\mu \lambda} \sqrt{\lambda\left(\frac{\alpha}{2} \mu-\lambda\right)\left(\frac{2}{\gamma}-1\right)}$ \\
\hline $\mathrm{F}$ & $\frac{\Pi}{\sqrt{6} \lambda}$ & $\frac{\Pi}{\sqrt{6} \mu}$ & $\frac{1}{\mu \lambda} \sqrt{\Pi \mu\left(\mu+\frac{\alpha}{2} \lambda\right)\left(1-\frac{\Pi}{6}\right)}$ & $\frac{1}{\mu \lambda} \sqrt{\Pi \lambda\left(\frac{\alpha}{2} \mu-\lambda\right)\left(1-\frac{\Pi}{6}\right)}$ \\
\hline
\end{tabular}

TABLE I: The real and physically meaningful critical points of the autonomous system (15). The parameter $\Pi$ is defined in (22).

\begin{tabular}{|c|c|c|c|c|}
\hline & Existence & Conditions for Stability & $\Omega_{D E}$ & $w_{\mathrm{DE}}$ \\
\hline $\bar{A}$ & all $\mu, \lambda, \alpha$ & unstable & 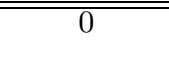 & undefined \\
\hline$\overline{\mathrm{B}}$ & all $\mu, \lambda, \alpha$ & unstable & 1 & 1 \\
\hline D1 & all $\mu, \lambda, \alpha$ & $\mu>-\alpha \lambda / 2$ & 1 & $-1-\frac{4 \mu^{2}}{3\left(4+\alpha^{2}\right)}$ \\
\hline $\mathrm{C} 2$ & $\lambda \geq \sqrt{\frac{3 \gamma}{4}\left(4+\alpha^{2}\right)}$ & $\lambda<\alpha \mu / 2$ & $\frac{3 \gamma}{4 \lambda^{2}}\left(4+\alpha^{2}\right)$ & $w_{M}$ \\
\hline D2 & $\lambda \leq \sqrt{\frac{3}{2}\left(4+\alpha^{2}\right)}$ & $\lambda<\min \left[\alpha \mu / 2, \sqrt{\frac{3 \gamma}{4}\left(4+\alpha^{2}\right)}\right]$ & 1 & $-1+\frac{4 \lambda^{2}}{3\left(4+\alpha^{2}\right)}$ \\
\hline $\mathrm{E}$ & $\alpha \mu-2 \lambda>0 ; 2 \mu+\alpha \lambda>0 ; \Pi \geq 3 \gamma$ & unstable & $3 \gamma / \Pi$ & $w_{M}$ \\
\hline $\mathrm{F}$ & $\begin{array}{c}\alpha \mu-2 \lambda>0 ; 0<\Pi<6 \\
2 \mu+\alpha \lambda<0\end{array}$ & $\begin{array}{l}\text { unstable } \\
\text { stable }\end{array}$ & 1 & $-1+\Pi / 3$ \\
\hline
\end{tabular}

TABLE II: The properties of the critical points of the autonomous system (15). The parameter $\Pi$ is defined in (22). Note that there are two disconnected regions in the parameter space for which point $F$ is physical.

$e_{3}$ and $e_{4}$ are always negative as the condition for existence gives

$$
6 \gamma^{2}\left(4+\alpha^{2}\right)+\lambda^{2}(2-9 \gamma) \leq \lambda^{2}(2-\gamma)
$$

Thus, the point is stable if $(2 \lambda-\alpha \mu)<0$, so unlike the quintom case (cf. point $\mathrm{T}$ in [8]), with a sufficiently large value of $\alpha \mu$ one obtains a stable scaling solution in this system.

\section{- Point D2}

This field-dominated solution is similar to $D 1$ in [18] and reduces to point $S$ in [8] when $\alpha=0$. The existence condition, $\lambda \leq \sqrt{\frac{3}{2}\left(4+\alpha^{2}\right)}$, is derived from the requirement that $y_{\phi c} \in \mathbb{R}$. The eigenvalues are

$$
\begin{gathered}
e_{1}=\frac{\lambda(2 \lambda-\alpha \mu)}{\left(4+\alpha^{2}\right)} \quad e_{2}=\frac{4 \lambda^{2}}{\left(4+\alpha^{2}\right)}-3 \gamma \\
e_{3,4}=\frac{2 \lambda^{2}}{\left(4+\alpha^{2}\right)}-3 \leq 0
\end{gathered}
$$

where the existence condition ensures that $e_{3,4}$ are negative. If the point is to be stable both $e_{1}$ and $e_{2}$ must be negative, which is satisfied if

$$
\lambda<\min \left[\alpha \mu / 2, \sqrt{\frac{3 \gamma}{4}\left(4+\alpha^{2}\right)}\right] .
$$

Once again, one can choose values of $\mu$ and $\alpha$ so that this point is stable, which is not the case in the standard quintom model.

\section{- Point $E$}

The points $E$ and $F$ are characterised by the fact that the potential terms for both the phantom and the quintessence field are non-zero. We mention that when $\alpha=0$ these points do not exist, since it is not possible to choose values for $\lambda$ and $\mu$ such that $y_{\phi c}, y_{\sigma c} \in \mathbb{R}$. Point $E$ is a scaling solution, with density parameter

$$
\Omega_{D E}=\frac{3 \gamma}{\lambda^{2} \mu^{2}}\left(\mu^{2}-\lambda^{2}+a \mu \lambda\right) .
$$

Following [18], we can rewrite this quantity in terms of an effective exponent $\Pi$ (which in the present case can be negative too, contrary to [18]) defined 
by

$$
\frac{1}{\Pi}=\frac{1}{\lambda^{2}}-\frac{1}{\mu^{2}}+\frac{\alpha}{\mu \lambda},
$$

which leads to the existence condition $\Pi \geq 3 \gamma$. Note that, as $\mu, \lambda \geq 0$,

$$
(\alpha \mu-2 \lambda)>0 \Rightarrow(2 \mu+\alpha \lambda)>0 .
$$

Thus, to ensure that $y_{\phi c}$ and $y_{\sigma c}$ are real, one must additionally impose $(\alpha \mu-2 \lambda)>0$. The eigenvalues are

$$
\begin{aligned}
e_{1,2} & =-\frac{3}{4}(2-\gamma) \\
& \times\left[1 \pm \sqrt{\frac{24 \gamma^{2} / \Pi+2-9 \gamma}{(2-\gamma)}}\right] \\
e_{3,4} & =-\frac{3}{4}(2-\gamma) \\
& \times\left[1 \pm \sqrt{1-\frac{8 \gamma(2 \mu+\alpha \lambda)(2 \lambda-\alpha \mu)}{\lambda \mu(2-\gamma)\left(4+\alpha^{2}\right)}}\right] .
\end{aligned}
$$

In terms of $\Pi$ the existence condition reads $\frac{24 \gamma^{2}}{\Pi}+$ $2-9 \gamma \leq(2-\gamma)$; substituting this into (24) leads to $e_{1,2} \leq-\frac{3}{4}(2-\gamma)(1 \pm 1) \leq 0$. However, looking at the fraction under the square root in (25), we observe that

$$
1-\frac{8 \gamma(2 \mu+\alpha \lambda)(2 \lambda-\alpha \mu)}{\lambda \mu(2-\gamma)\left(4+\alpha^{2}\right)}>1 .
$$

Thus, either $e_{3}$ or $e_{4}$ must be positive, and therefore the critical point is unstable.

\section{- Point $F$}

In this (field-dominated) point we observe the presence of square-root terms in $y_{\phi c}$ and $y_{\sigma c}$. Thus, for these values to be real we require

$$
\begin{aligned}
& \Pi\left(1-\frac{\Pi}{6}\right)(2 \mu+\alpha \lambda)>0, \\
& \Pi\left(1-\frac{\Pi}{6}\right)(\alpha \mu-2 \lambda)>0 .
\end{aligned}
$$

Using (22) and (23), and noting that

$$
(2 \mu+\alpha \lambda)<0 \Rightarrow(\alpha \mu-2 \lambda)<0,
$$

and

$$
\begin{aligned}
(2 \mu+\alpha \lambda)<0 & \Rightarrow \mu^{2}-\lambda^{2}+a \mu \lambda<0 \\
& \Rightarrow \Pi<0,
\end{aligned}
$$

we conclude that there exist two possible cases:

(a) $\alpha \mu-2 \lambda>0 ; \quad 0<\Pi<6$,

(b) $2 \mu+\alpha \lambda<0$.
The eigenvalues read

$$
\begin{aligned}
e_{1} & =-3\left(1-\frac{\Pi}{6}\right) \\
e_{2} & =\Pi-3 \gamma \\
e_{3,4} & =-\frac{3}{2}\left(1-\frac{\Pi}{6}\right) \\
& \times\left[1 \pm \sqrt{1-\frac{4 \Pi}{3 \lambda \mu} \frac{(2 \mu+\alpha \lambda)(2 \lambda-\alpha \mu)}{\left(4+\alpha^{2}\right)\left(1-\frac{\Pi}{6}\right)}}\right] .
\end{aligned}
$$

In case (a), $\left(1-\frac{\Pi}{6}\right)>0$, thus $e_{1}<0$. However, in a similar way to the analysis of point $E$, the term under the square root in the expression for $e_{3,4}$ is greater than 1 so either $e_{3}$ or $e_{4}$ is positive and the point is unstable. In case (b), the term under the square root is less than 1 so $e_{3,4}<0 . e_{2}<0$ is trivially satisfied [using (27)] and, again, $\left(1-\frac{\Pi}{6}\right)>0$, therefore $e_{1}<0$. Thus, the point is stable whenever the condition (29) is satisfied. Finally, we mention that this separation of the parameter space is a novel feature of the scenario at hand, which is not present in the corresponding case of two canonical fields [18].

The stability regions for the points are plotted in Fig. 1. In the standard quintom case $(\alpha=0)$, there is only one stable critical point that exists for all values of $\mu$ and $\lambda$, corresponding to the phantom point D1. In the case $\alpha<$ 0 the parameter space is fractured, thus, although there is a stable point with $w_{\mathrm{DE}}<-1$ at each parameter-space region, the precise value of $w_{\mathrm{DE}}$ for particular values of $\mu$ and $\lambda$ depends on whether point $D 1$ or $F$ is stable. In the case where $\alpha>0$, the phantom dominated point $D 1$ is always stable. It is interesting, however, that there are also regions where C2 (a scaling solution) and D2 (a quintessence-dominated solution), each with $w_{\mathrm{DE}}>-1$, are stable. In these regions the initial conditions will determine the cosmological evolution.

As we have seen, point $F$ exists in two disconnected regions in the parameter space: a stable region where $\alpha<0$ and an unstable region where $\alpha>0$. This can be understood as a fracture of the unstable regions in the parameter space, depicted in Fig. 2. In the standard quintom case, there are two overlapping regions corresponding to C2 and D2. When $\alpha \mu-2 \lambda>0$ the points $E$ and $F$ are present and encroach upon this region. As one might expect, in both cases, when the exponent of the quintessence field is large, the scaling solution does exist (although it is not always stable).

\section{COSMOLOGICAL IMPLICATIONS}

Having performed the complete phase-space analysis of the model, we can discuss the corresponding cosmological behaviour. A general remark is that the phenomenology is different from both the standard $(\alpha=0)$ quintom 


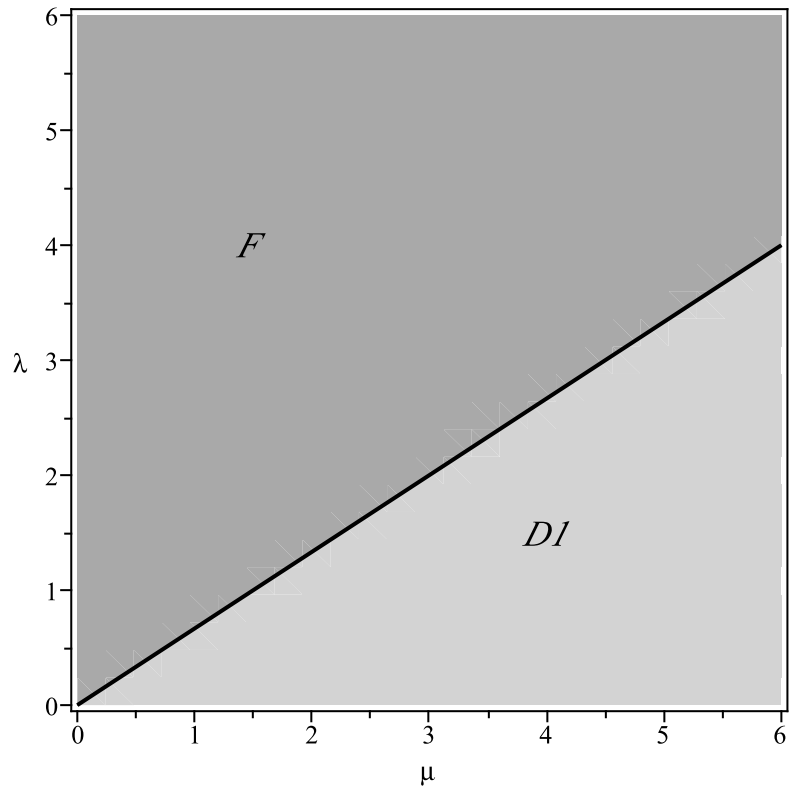

(a)

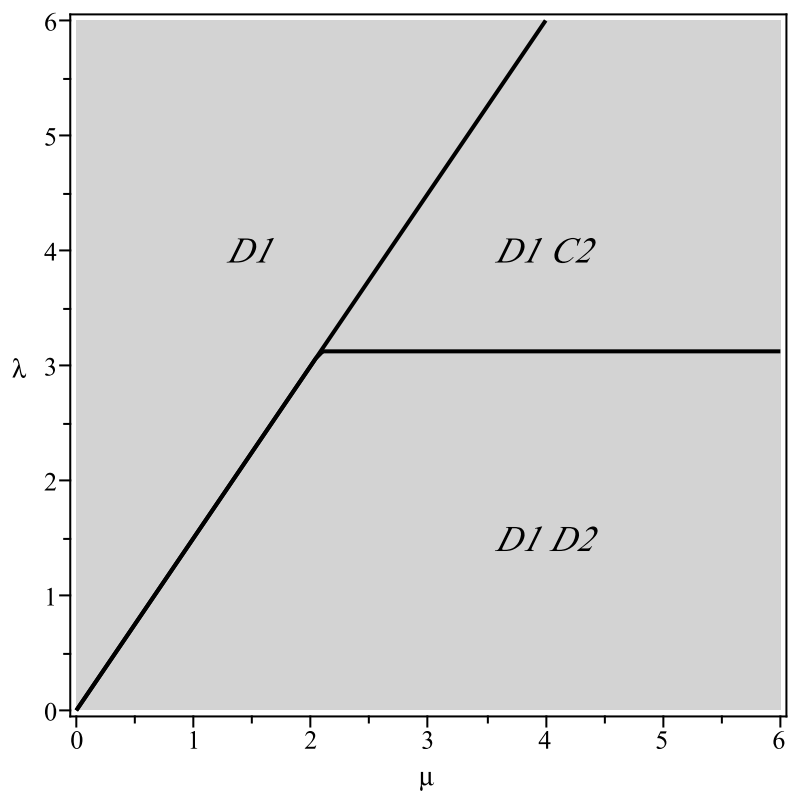

(b)

FIG. 1: The parameter space showing the stability of the critical points for (a) $\alpha=-3$ and (b) $\alpha=3$, with $\gamma=1$. The light shaded area indicates the region where $D 1$ is stable and the dark shaded area indicates the region where $F$ is stable. There is a stable point with $w_{\mathrm{DE}}<-1$ at each region in the parameter space. The area marked in graph (b) indicates the region where $C_{2} 2$ and $D 2$ are stable.

scenario [8, 9] and from the case of quintessence with mixed kinetic terms [18]. In particular, there are latetime solutions describing both quintessence and phantom dominated universes, with the crucial observable quantity being the dark energy equation-of-state parameter

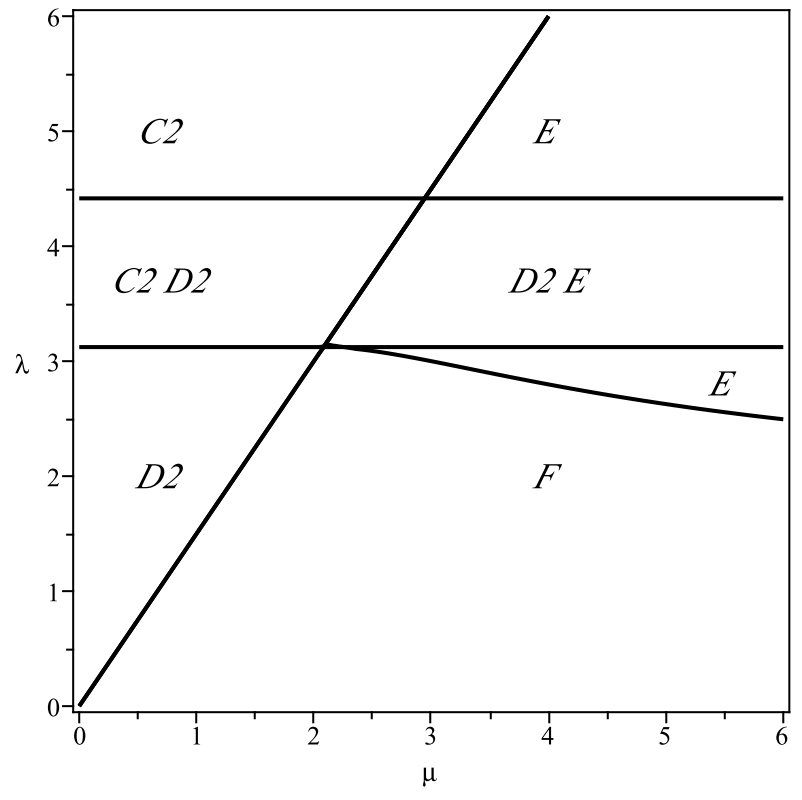

FIG. 2: Regions in the parameter space where the critical points exist but are unstable for $\alpha=3$ (with $\gamma=1$ ).

$w_{\mathrm{DE}}$ (which is greater than -1 in the former case and less than -1 in the latter).

The points $A, B$ and $E$ are unstable, and thus cannot be late-time solutions of the Universe. $A$ corresponds to a matter-dominated universe, while $B$ represents a dark energy-dominated one, with the stiff value $w_{\mathrm{DE}}=1 . E$ is a scaling solution. Finally, in one of the two disconnected areas in which $F$ is physical, when $\alpha \mu-2 \lambda>0$ and $0<\Pi<6$, which corresponds to $w_{\mathrm{DE}}>-1$, the point is always unstable.

As usual, the stable critical points can be classified as field-dominated $\left(\Omega_{D E}=1\right)$ or scaling solutions $\left(w_{\mathrm{DE}}=0\right.$ in the presence of matter). Since these features are incompatible with observations, we deduce that the latetime behaviour of the scenario considered here cannot provide a satisfactory description of the Universe at the present time. However, the fact that dark energy has only recently begun to dominate the energy density, after an extended matter-dominated era, suggests that the Universe may have yet to reach an attractor solution. In this case, one can consider our Universe as evolving towards a field dominant attractor, although the problem with initial conditions is still an issue [26].

In order to treat the coincidence problem one must explain why the present dark energy and matter density parameters are of the same order of magnitude. Alternatively, one can ask why dark energy has only recently become dynamically important, which is a problem of initial conditions. This can be alleviated if $\rho_{D E}$ scales with the dominant matter component, as the discrepancy between the initial energy densities of the various components of the Universe does not have to be so large.

In contrast to the simple quintom scenario with $\alpha=$ 
0 , there is a stable scaling solution in this model, viz. point C2, which is an extension of the unstable point $\mathrm{T}$ in [8]. However, as the present model stands, there is no mechanism to effect a transition between the scaling regime and field-dominant solution without fine-tuning the initial conditions.

Another novel feature of the extended quintom scenario is a stable field-dominated late-time solution with $w_{\mathrm{DE}}>-1$ (point D2) which is characterised by the fact that the contribution of the potential of the phantom field to the energy density is zero. Like $C 2$, this is an extension of an unstable point in the simple quintom scenario. The deviation from $w_{\mathrm{DE}}=-1$ is $4 \lambda^{2} /\left[3\left(4+\alpha^{2}\right)\right]$ so one requires either the coupling to be large or the quintessence potential to be sufficiently flat for this solution to be compatible with observations.

Let us make an important comment concerning C2 and D2. As can be seen in Table I, both points have $x_{\sigma c}>0$ in the regions in which they are stable (we consider positive potential exponents $\lambda, \mu>0$ ). However, since phantom fields generally do not roll down their potential slopes, one would normally expect that in a stable late-time solution, the rate of change of the phantom field, that is $x_{\sigma c}$, should be negative (in a stationary solution) or zero (in a static solution). Indeed, for the simple quintom case $(\alpha=0)$ we do obtain $x_{\sigma c}=0$. Interestingly enough, the positive coupling of the kinetic terms of the two fields makes the quintessence field drag the phantom field down its potential (i.e. the opposite to "normal" phantom behaviour). This possibility is a novel feature of the present scenario.

The point $D 1$ is the analogue of $D 2$ and is stable for the larger part of the parameter space (it is always stable for $\alpha \geq 0$, while for $\alpha<0$ it can still be stable for $\mu>$ $-\alpha \lambda / 2) . w_{\mathrm{DE}}$ depends only on the values of $\mu$ and $\alpha$ and approaches -1 if the potential is flat or the coupling is large, in a similar manner to the quintessence-dominated point, D2. Furthermore, it is interesting to note in this case there is a situation similar to the one described in the previous paragraph. In particular, for $\alpha<0$ we observe that $x_{\phi c}<0$, so, contrary to expectations, there is a stable late-time solution where the quintessence field rolls up its potential slope. Again, this is due to the coupling of the kinetic terms: the phantom field feeds the upward rolling of the quintessence field.

From the discussion of the above two paragraphs we deduce that the kinetic coupling leads to new stable solutions. Additionally, it could lead to a very interesting evolution towards these late-time solutions. In particular, if the kinetic terms are initially small, the effect of the coupling is downgraded, and the fields can move away from these stable points. With the increase of their kinetic energies, the coupling becomes more important and they will start to move towards them.

When point $D 1$ is unstable, the critical point $F$, in one of the two disconnected areas for which it is physical, viz. $2 \mu+\alpha \lambda<0$, is stable. It corresponds to a universe completely dominated by dark energy, behaving like a phantom, with the EOS determined by the parameter $\Pi$. In this case, the quintessence and phantom fields combine to give a solution with $w_{\mathrm{DE}}<-1$. This is the analogue of point $F$ in [18] and, like the unstable point $E$, does not exist in the standard quintom case.

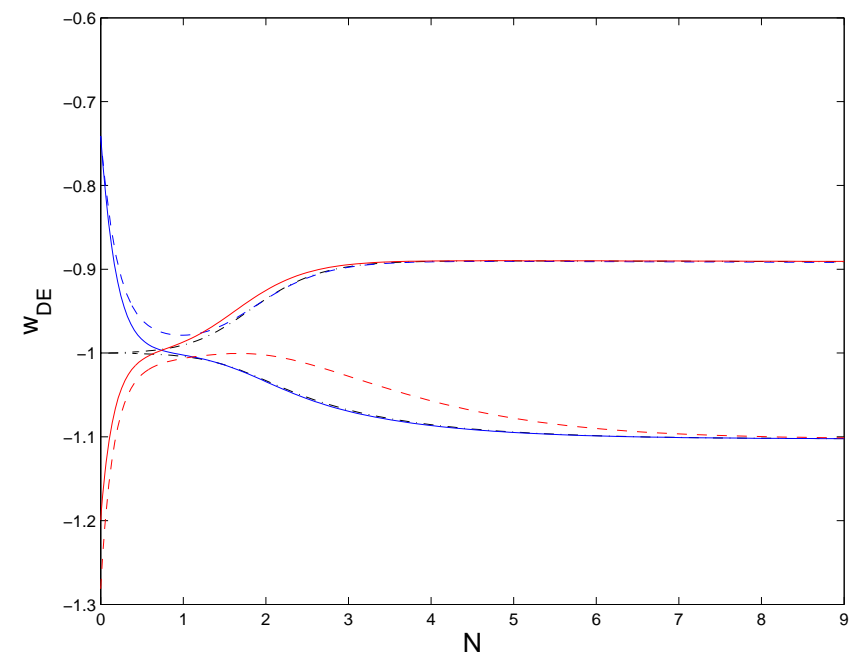

(a)

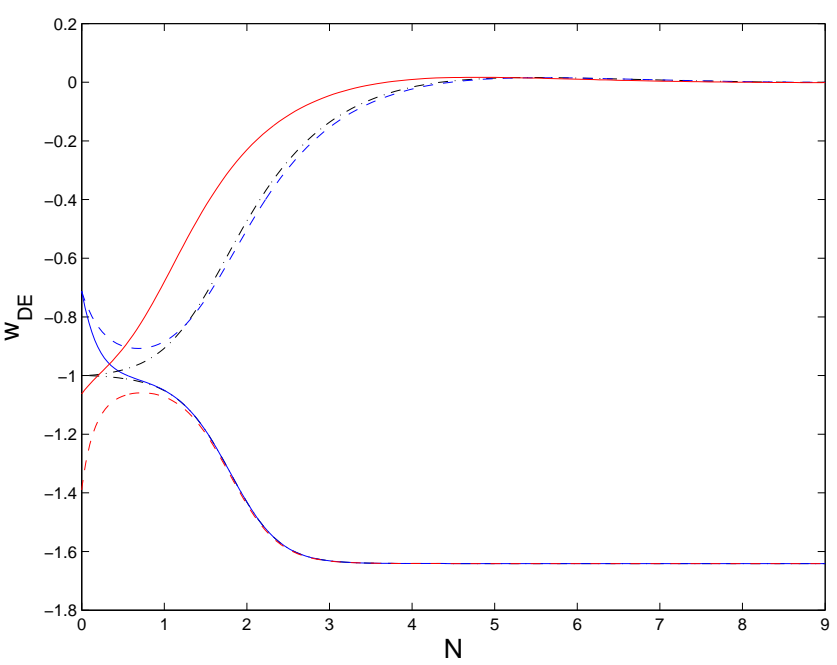

(b)

FIG. 3: Numerical integration of the system with (a) $\mu=1$, $\lambda=1, \alpha=3$ and (b) $\mu=2.5, \lambda=3.5, \alpha=3$ with initial conditions chosen so that the matter field (with $w_{M}=0$ ) initially dominates. A variety of plots with different initial conditions are shown: the colour-coding is for visual clarity. In case (a) points D1 and D2 are stable; one can see that there are solutions that cross the phantom divide to reach the latetime attractors. A similar situation is seen in case (b) where D1 and C2 are stable.

In general, if a cosmological model possesses more than one stable critical point, amongst the possible late-time solutions it will reach the one in the basin of attraction of which it lies. Thus, the specific cosmological evolution depends heavily on the initial conditions. In our scenario, there are regions of the parameter space where there is 
both a stable quintessence-like and a stable phantomlike critical point. Therefore, the EOS can always remain in the quintessence or the phantom regime, cross the phantom divide from below to above, or cross the phantom divide from above to below, with the last possibility mildly favoured by observations. It must be noted, however, that in the absence of constraints on the coupling $\alpha$ and the potential exponents, a late-time solution with $w_{\mathrm{DE}}<-1$ seems likely as there is a stable phantomlike critical point in every region of the parameter space. This fact is an advantage of the model.

As we know, the standard quintom model [8 10] exhibits only one (phantom-like) stable late-time solution, and in order to acquire the simultaneous existence of one quintessence and one phantom stable late-time solutions, one has to extend to significantly generalized quintom models 24]. On the other hand, the model at hand presents this behavior more economically. Furthermore, note that the extended scenario of this work exhibits a novel situation, viz. the presence of more than one stable attractor (points C2 and D2) corresponding to quintessence-like solutions. Thus, according to the specific parameter values, and for suitable initial conditions, the late-time behaviour of the Universe can be described by one of the quintessence-like states, with $D 2$ possessing a more physical EOS compared to C2 (see Table II).

In order to present the late-time behaviour transparently, in Fig. 3 we depict the results of a numerical integration for two choices of parameter values. In graph (a) the phantom stable point is $D 1$ and the quintessence one is D2, while in graph (b) the phantom stable point is also $D 1$ but the quintessence one is $\mathrm{C} 2$. If the system avoids the stable phantom solution, it will tend toward one of the two quintessence-like solutions, depending on the values of the parameters, $\alpha, \mu$ and $\lambda$.

We have to mention that for a particular subclass of evolutionary trajectories, depending on the parameter values and initial conditions, the dark energy EOS becomes infinite at a finite time $t_{*}$. However, this divergence in $w_{\mathrm{DE}}$ is not accompanied by a divergence in the scale factor, its time-derivative or in the dark energy density and pressure, but is an expected result arising from the nullification of $\rho_{D E}$, possible in all phantom models. Thus, technically, it does not correspond to the Big Rip discussed in the literature 27], but rather to the new singularity family discussed in [28]. One could consider only those models in which the total energy density remains positive (where the potential is sufficiently positive or the phantom kinetic energy not too large), to avoid the corresponding energy condition violation. Finally, note that while a negative coupling value $\alpha$ facilitates the nullification of $\rho_{D E}$, a positive $\alpha$ obstructs it.

As one can observe in Fig. 3, the dark energy EOS can change significantly before the system finally reaches the critical points. It is therefore of great interest to investigate whether the situation described above is compatible with the stringent bounds provided by cosmological observations. To this end, in Fig. 4 we replot the evolution

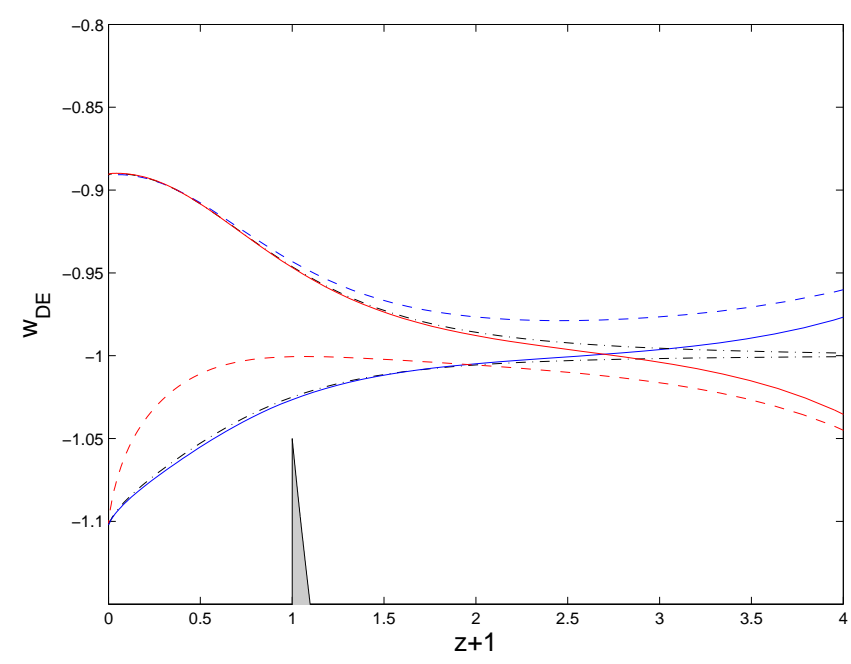

(a)

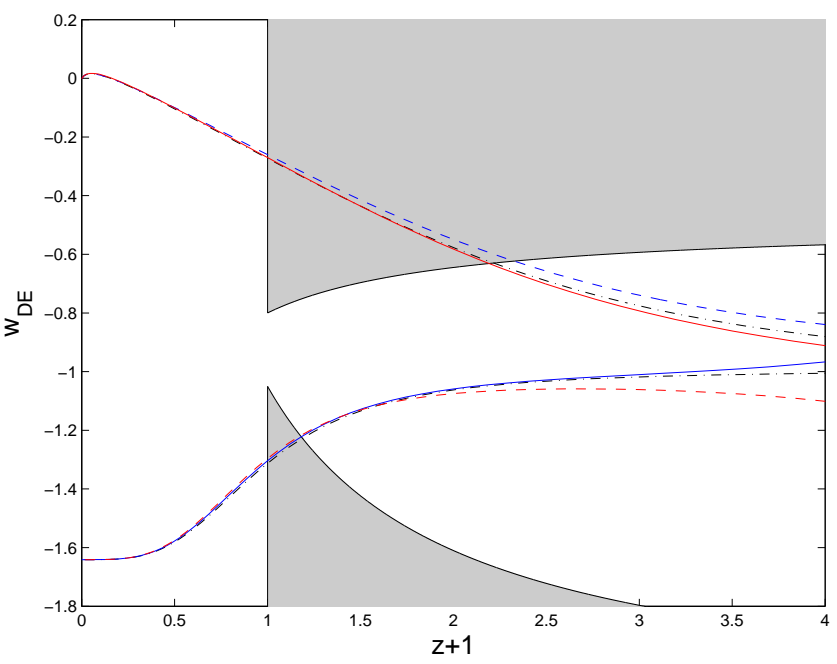

(b)

FIG. 4: The evolution of the dark energy EOS with respect to redshift $\left[z+1=a\left(t_{0}\right) / a(t)\right]$. Parameter values, initial conditions and colour-coding are as in Fig. $4 z=0$ is chosen to correspond to the point at which $\Omega_{D E}=0.728$. Shaded regions are excluded by Wilkinson Microwave Anisotropy Probe $7 \mathrm{yr}$ data $+\mathrm{BAO}+H_{0}+\mathrm{SN}$ at $68 \%$ confidence level [29]. Using the parameterisation $w_{\mathrm{DE}}=w_{0}+w_{a} z /(z+1)$, the limits are $w_{0}=0.93 \pm 0.13$ and $w_{a}=-0.41_{-0.71}^{+0.72}$.

of $w_{\mathrm{DE}}$ of Fig. 3, using the redshift $z\left[z+1 \equiv a\left(t_{0}\right) / a(t)\right]$ as the independent variable, presenting also the observationally excluded regions from [29], up to $z=3$. In the upper graph, both potentials are relatively flat and the values of $w_{\mathrm{DE}}$ at the critical points $D 1$ and $D_{2}$ are close to -1 . This means that, despite the range of behaviour exhibited for different initial conditions, the EOS does not deviate much from -1 when the trajectories are approaching the attractors at recent times. These features make the evolutions of Fig. 4(a) compatible with observations.

The case in Fig. 4(b) is quite different. As mentioned 
previously, it can be seen that the scaling point $C 2$ is ruled out as a late-time solution. Also, for both $C 2$ and $D 1$ to be stable, $\lambda$ needs to be larger, leading to a more negative EOS for D1. As the trajectories start to converge on this value, $w_{\mathrm{DE}}$ evolves more quickly than Fig. 4(a), leading to an EOS that is disfavoured by observations at $z \approx 0$.

The behaviour of Fig. 4 is fairly typical. In the case where the dark energy contribution to the energy content of the Universe is initially negligible, the value $\Omega_{D E} \approx$ 0.7 is obtained only when the solutions start to converge to the critical points. Thus, if the value of $w_{\mathrm{DE}}$ at the critical points is closer to -1 , the variation in the EOS is smaller at recent times, and closer to the observational values.

In order to perform a complete comparison with the data, it is necessary to take into account the effect of dark energy perturbations. In particular, the late-time integrated Sachs-Wolfe effect, which affects the CMB on large angular scales, is sensitive to the presence of dark energy perturbations 22]. Perturbations in the standard quintom model have been investigated in 30, where it was found that the parameter space that allowed the EOS to cross the phantom divide was enlarged when the perturbations were included. As the present scenario exhibits behaviour similar to simple quintessence and quintom models, we expect that many features of these scenarios will be retained when the perturbations are taken into account. However, as we have seen, an important difference between the coupled and uncoupled cases is the presence of a source term proportional to $\alpha$ that can drag the quintessence (phantom) field up (down) its potential. This would introduce extra terms into the perturbation equations which could lead to observable effects. Another interesting possibility is that the behaviour of isocurvature perturbations will be affected by the transfer of energy between the two fields. The full analysis of the perturbations in the model at hand is beyond the scope of the current investigation and will be treated in a future work 31.

\section{CONCLUSIONS}

We have considered an extension of the quintom model of dark energy in which the kinetic terms of the phantom and the canonical scalar field are coupled by a term in the Lagrangian of the form $\frac{\alpha}{2} g^{\mu \nu} \partial_{\mu} \phi \partial_{\nu} \sigma$. In order to study the asymptotic behaviour of the model and to facilitate comparison both with the standard quintom scenario and the two-field quintessence model with mixed kinetic terms, we have performed a phase-space analysis of the corresponding dynamical system.

We find that the kinetic interaction allows for the possibility of stable critical points similar to those found in quintessence scenarios, including field-dominated solutions with $w_{\mathrm{DE}}>-1$ and solutions displaying scaling behaviour. Additionally, there exist two new criti- cal points $E$ and $F$ that are not present in the simple phantom model, the latter of which is analogous to that responsible for the phenomenon of assisted quintessence in the case with two canonical fields. Here, however, the combined effect of the fields is to give phantom-like behaviour, with $w_{\mathrm{DE}}<-1$. As well as this, there is an extension of the phantom-dominated asymptotic solution in the standard quintom model, which is stable for a large region of the parameter space. In all, there is a stable solution with $w_{\mathrm{DE}}<-1$ and $\Omega_{D E}=1$ in every part of the parameter space, even when the quintessence-like points are stable. We have discussed the cosmological implications of these results in Sec. IV]

A characteristic feature of the presence of the kinetic coupling is the presence of stable solutions in which the quintessence field can be dragged up its potential, in the opposite direction to the standard case. This occurs when the coupling is negative, where the quintessence equation of motion acquires a positive source term. Similarly, for a positive coupling the phantom-field equation of motion obtains a negative source term, which causes the field to roll down its potential. This could affect the evolution of the dark energy perturbations and lead to observational differences between the kinetically coupled quintom scenario and other models, offering a relatively safe signature.

The use of canonical and phantom fields to drive the acceleration of the Universe in phenomenological models is not necessarily representative of the fundamental mechanism behind dark energy, in which non-trivial features such as non-canonical kinetic terms and kinetic couplings may be important. The scenario of the present work is interesting as the kinetic coupling gives rise to behaviour different from that of the standard quintom model. In particular, both quintessence-like and phantom-like late-time solutions, as well as solutions that cross the phantom divide $w_{\mathrm{DE}}=-1$ during the evolution, are possible. Thus, such scenarios could be a candidate for the description of dark energy.

\section{Acknowledgments}

We would like to thank Carsten van de Bruck for useful discussions and for comments on the manuscript. JW is supported by EPSRC.

\section{Appendix: The linearised perturbation matrix}

The $4 \times 4$ matrix $\mathbf{Q}$ of the linearised perturbation equations of the system (15) reads:

$$
\mathbf{Q}=\left[\begin{array}{llll}
Q_{11} & Q_{12} & Q_{13} & Q_{14} \\
Q_{21} & Q_{22} & Q_{23} & Q_{24} \\
Q_{31} & Q_{32} & Q_{33} & Q_{34} \\
Q_{41} & Q_{42} & Q_{43} & Q_{44}
\end{array}\right]
$$


with

$$
\begin{aligned}
Q_{11} & =-3+\frac{3}{2} x_{\phi c}(2-\gamma)\left(2 x_{\phi c}+\alpha x_{\sigma c}\right)+T_{c} \\
Q_{12} & =\frac{3}{2} x_{\phi c}(2-\gamma)\left(-2 x_{\sigma c}+\alpha x_{\phi c}\right) \\
Q_{13} & =y_{\phi c}\left[\frac{4 \sqrt{6} \lambda}{\left(4+\alpha^{2}\right)}-3 \gamma x_{\phi c}\right] \\
Q_{14} & =y_{\sigma c}\left[\frac{2 \alpha \sqrt{6} \mu}{\left(4+\alpha^{2}\right)}-3 \gamma x_{\phi c}\right] \\
Q_{21} & =\frac{3}{2} x_{\sigma c}(2-\gamma)\left(2 x_{\phi c}+\alpha x_{\sigma c}\right), \\
Q_{22} & =-3+\frac{3}{2} x_{\sigma c}(2-\gamma)\left(-2 x_{\sigma c}+\alpha x_{\phi c}\right)+T_{c}, \\
Q_{23} & =y_{\phi c}\left[\frac{2 \alpha \sqrt{6} \lambda}{\left(4+\alpha^{2}\right)}-3 \gamma x_{\sigma c}\right] \\
Q_{24} & =-y_{\sigma c}\left[\frac{4 \sqrt{6} \mu}{\left(4+\alpha^{2}\right)}+3 \gamma x_{\sigma c}\right] \\
Q_{31} & =y_{\phi c}\left[\frac{3}{2}(2-\gamma)\left(2 x_{\phi c}+\alpha x_{\sigma c}\right)-\sqrt{\frac{3}{2}} \lambda\right] \\
Q_{32} & =\frac{3}{2} y_{\phi c}(2-\gamma)\left(-2 x_{\sigma c}+\alpha x_{\phi c}\right),
\end{aligned}
$$

$$
\begin{aligned}
Q_{33} & =-\sqrt{\frac{3}{2}} \lambda x_{\phi c}-3 \gamma y_{\phi c}^{2}+T_{c} \\
Q_{34} & =-3 \gamma y_{\phi c} y_{\sigma c}
\end{aligned}
$$$$
\begin{aligned}
Q_{41} & =\frac{3}{2} y_{\sigma c}(2-\gamma)\left(2 x_{\phi c}+\alpha x_{\sigma c}\right), \\
Q_{42} & =y_{\sigma c}\left[\frac{3}{2}(2-\gamma)\left(-2 x_{\sigma c}+\alpha x_{\phi c}\right)-\sqrt{\frac{3}{2}} \mu\right], \\
Q_{43} & =-3 \gamma y_{\phi c} y_{\sigma c} \\
Q_{44} & =-\sqrt{\frac{3}{2}} \mu x_{\sigma c}-3 \gamma y_{\sigma c}^{2}+T_{c}
\end{aligned}
$$

where

$$
\begin{aligned}
T_{c}= & \frac{3}{2}\left[2 x_{\phi c}^{2}-2 x_{\sigma c}^{2}+2 \alpha x_{\phi c} x_{\sigma c}+\right. \\
& \left.+\gamma\left(1-x_{\phi c}^{2}+x_{\sigma c}^{2}-\alpha x_{\phi c} x_{\sigma c}-y_{\phi c}^{2}-y_{\sigma c}^{2}\right)\right] .
\end{aligned}
$$

[1] A. G. Riess et al. [Supernova Search Team Collaboration], Astron. J. 116, 1009 (1998); S. Perlmutter et al. [Supernova Cosmology Project Collaboration], Astrophys. J. 517, 565 (1999).

[2] C. L. Bennett et al., Astrophys. J. Suppl. 148, 1 (2003); E. Komatsu et al. [WMAP Collaboration], Astrophys. J. Suppl. 180, 330 (2009) arXiv:0803.0547 [astro-ph]].

[3] M. Tegmark et al. [SDSS Collaboration], Phys. Rev. D 69, 103501 (2004).

[4] S. W. Allen, et al., Mon. Not. Roy. Astron. Soc. 353, 457 (2004).

[5] V. Sahni and A. Starobinsky, Int. J. Mod. Phy. D 9, 373 (2000); P. J. Peebles and B. Ratra, Rev. Mod. Phys. 75, 559 (2003).

[6] B. Ratra and P. J. E. Peebles, Phys. Rev. D 37, 3406 (1988); C. Wetterich, Nucl. Phys. B 302, 668 (1988); A. R. Liddle and R. J. Scherrer, Phys. Rev. D 59, 023509 (1998); I. Zlatev, L. M. Wang and P. J. Steinhardt, Phys. Rev. Lett. 82, 896 (1999); Z. K. Guo, N. Ohta and Y. Z. Zhang, Mod. Phys. Lett. A 22, 883 (2007); S. Dutta, E. N. Saridakis and R. J. Scherrer, Phys. Rev. D 79, 103005 (2009).

[7] R. R. Caldwell, Phys. Lett. B 545, 23 (2002); R. R. Caldwell, M. Kamionkowski and N. N. Weinberg, Phys. Rev. Lett. 91, 071301 (2003); S. Nojiri and S. D. Odintsov, Phys. Lett. B 562, 147 (2003); V. K. Onemli and R. P. Woodard, Phys. Rev. D 70, 107301 (2004); M. R. Setare and E. N. Saridakis, JCAP 0903, 002 (2009); E. N. Saridakis, Nucl. Phys. B 819, 116 (2009).

[8] Z. K. Guo, Y. S. Piao, X. M. Zhang and Y. Z. Zhang, Phys. Lett. B 608, 177 (2005).

[9] B. Feng, X. L. Wang and X. M. Zhang, Phys. Lett. B
607, 35 (2005); M.-Z Li, B. Feng, X.-M Zhang, JCAP, 0512, 002 (2005); B. Feng, M. Li, Y.-S. Piao and X. Zhang, Phys. Lett. B 634, 101 (2006); W. Zhao and Y. Zhang, Phys. Rev. D 73, 123509 (2006); M. R. Setare and E. N. Saridakis, Phys. Lett. B 668, 177 (2008).

[10] Y. F. Cai, E. N. Saridakis, M. R. Setare and J. Q. Xia, Phys. Rep. 4, 001 (2010)

[11] C. Wetterich, Astron. Astrophys. 301, 321 (1995) L. Amendola, Phys. Rev. D 60, 043501 (1999); L. Amendola, Phys. Rev. D 62, 043511 (2000); D. TocchiniValentini and L. Amendola, Phys. Rev. D 65, 063508 (2002); G. R. Farrar and P. J. E. Peebles, Astrophys. J. 604, 1 (2004); T. Gonzalez, G. Leon and I. Quiros, Class. Quant. Grav. 23, 3165 (2006). A. W. Brookfield, C. van de Bruck and L. M. .H Hall, Phys. Rev. D 77, 043006 (2008).

[12] Z. K. Guo, R. G. Cai and Y. Z. Zhang, JCAP 0505, 002 (2005); R. Curbelo, T. Gonzalez, G. Leon and I. Quiros, Class. Quant. Grav. 23, 1585 (2006); X. m. Chen, Y. g. Gong and E. N. Saridakis, JCAP 0904, 001 (2009); E. N. Saridakis and S. V. Sushkov, Phys. Rev. D 81, 083510 (2010).

[13] M. Tegmark et al., Phys. Rev. D69, 103501 (2004); U. Alam, V. Sahni, T. D. Saini, and A. A. Starobinsky, Mon. Not. Roy. Astron. Soc. 354, 275 (2004); P. S. Corasaniti, M. Kunz, D. Parkinson, E. J. Copeland, and B. A. Bassett, Phys. Rev. D70, 083006 (2004).

[14] H. Kodama and M. Sasaki, Prog. Theor. Phys. Suppl. 78, 1 (1984); A. A. Starobinsky, JETP Lett. 42 (1985) 152 [Pisma Zh. Eksp. Teor. Fiz. 42, 124 (1985)]; D. Langlois, Phys. Rev. D 59, 123512 (1999).

[15] A. R. Liddle, A. Mazumdar and F. E. Schunck, Phys. 
Rev. D 58, 061301(R) (1998); K. A. Malik and D. Wands, Phys. Rev. D 59, 123501 (1999); A. A. Coley and R. J. van den Hoogen, Phys. Rev. D 62, 023517 (2000); G. Calcagni and A. R. Liddle, Phys. Rev. D 77, 023522 (2008).

[16] T. Barreiro, E. J. Copeland and N. J. Nunes, Phys. Rev. D 61, 127301 (2000).

[17] D. Blais and D. Polarski, Phys. Rev. D 70, 084008 (2004); S. A. Kim, A. R. Liddle and S. Tsujikawa, Phys. Rev. D 72, 043506 (2005); S. Tsujikawa, Phys. Rev. D 73, 103504 (2006); J. Ohashi and S. Tsujikawa, Phys. Rev. D 80, 103513 (2009).

[18] C. van de Bruck and J. M. Weller, Phys. Rev. D 80 123014 (2009).

[19] L. P. Chimento, M. Forte, R. Lazkoz and M. G. Richarte, Phys. Rev. D 79, 043502 (2009); S. Sur, arXiv:0902.1186 [astro-ph.CO]].

[20] D. Langlois and S. Renaux-Petel, JCAP 0804017 (2008).

[21] P.G. Ferreira, M. Joyce, Phys. Rev. Lett. 79, 4740 (1997); Y.G. Gong, A. Wang, Y.Z. Zhang, Phys. Lett. B 636, 286 (2006).

[22] E.J. Copeland, M. Sami, S. Tsujikawa, Int. J. Mod. Phys. D 15, 1753 (2006).

[23] E. J. Copeland, A. R. Liddle and D. Wands, Phys. Rev.
D 57, 4686 (1998).

[24] M. R. Setare and E. N. Saridakis, JCAP 0809, 026 (2008).

[25] R. J. Scherrer and A. A. Sen, Phys. Rev. D 77, 083515 (2008); R. J. Scherrer and A. A. Sen, Phys. Rev. D 78, 067303 (2008); M. R. Setare and E. N. Saridakis, Phys. Rev. D 79, 043005 (2009).

[26] U. J. Lopes Franca and R. Rosenfeld, JHEP 0210, 015 (2002).

[27] R. R. Caldwell, M. Kamionkowski and N. N. Weinberg, Phys. Rev. Lett. 91, 071301 (2003); P. F. GonzalezDiaz, Phys. Rev. D 68, 021303(R) (2003); R. Kallosh, J. Kratochvil, A. Linde, E. Linder and M. Shmakova, JCAP 0310, 015 (2003); G. W. Gibbons, arXiv:hep-th/0302199.

[28] K. Bamba, S. Nojiri and S. D. Odintsov, JCAP 0810, 045 (2008); M. R. Setare and E. N. Saridakis, Phys. Lett. B 671, 331 (2009); S. Capozziello, M. De Laurentis, S. Nojiri and S. D. Odintsov, Phys. Rev. D 79, 124007 (2009).

[29] E. Komatsu, et. al., arXiv:1001.4538 [astro-ph.CO].

[30] G.-B. Zhao, J.-Q. Xia, M. Li, B. Feng and X. Zhang, Phys. Rev. D 72, 123515 (2005).

[31] S. Dutta, E. N. Saridakis and J. M. Weller, in preparation. 